\title{
On the Nonlinear Dynamics of Turbulent Thermals in the Shear Flow
}

\author{
L. Kh. Ingel
}

The known nonlinear integral model of a turbulent thermal is generalized to the case of the presence of the horizontal component of its motion relative to the surrounding medium (for example, the floating-up of a thermal in a shear flow). In addition, the possibility of the presence of a heat (buoyancy) source in a thermal is considered. In comparison with the author's previous work, a solution is investigated for the case of unstable background stratification of the medium. The problem is solved in terms of quadratures. The asymptotics of the solution at large time intervals is analyzed. The solution describes, in particular, the nonlinear effect of the interaction of the horizontal and vertical components of the thermal motion, since each of the components affects the rate of entrainment of the surrounding medium, i. e., the growth rate of the thermal size and, hence, its mobility.

Keywords: convection, thermals, turbulence, integral models, shear flows, unstable stratification, nonlinear dynamics, analytical solutions

Received September 05, 2018

Accepted October 26, 2018

This work was supported by the Basic Research Program No. 56 of the Presidium of the Russian Academy of Sciences.

Lev Kh. Ingel

lev.ingel@gmail.com

Research and Production Association Typhoon

ul. Pobedy 4, Obninsk, 249038 Russia

Obukhov Institute of Atmospheric Physics, Russian Academy of Sciences

Pyzhevskii per. 3, Moscow, 119017 Russia

Interdepartment Center of Analytical Research in Physics,

Chemistry and Biology at the Presidium of the Russian Academy of Sciences

ul. Profsoyuznaya 65 (6), Moscow, 117997 Russia 


\section{Introduction}

In a recent paper by the author [1] a theoretical study was made of the problem of the motion of a turbulent thermal in a horizontal shear flow using the well-known model of turbulent convection from localized sources of buoyancy and (or) momentum, which act for a short time isolated thermals (see, for example, [2-7] and references therein). In such a model, the region of perturbation (thermal) is approximately represented in the form of an ascending (or descending, depending on the sign of perturbation) "bubble" or vortex ring of variable volume and mass. The volume of the thermal increases gradually as it captures the adjacent volumes of the environment (for brevity, the term "entrainment" is often used). For the thermal, a system of equations of the balance of mass, momentum and buoyancy is written. For example (see [2, Section 6.3.2], [6]), a system of equations is considered which, up to notation, has the form

$$
\frac{d}{d t} R^{3}=3 \beta R^{2} w, \quad \frac{d}{d t}\left(R^{3} w\right)=\beta_{1} R^{3} b, \quad \frac{d}{d t}\left(R^{3} b\right)=-N^{2} R^{3} w .
$$

Here $R, w$ and $b$ are the unknown radius, vertical velocity and specific buoyancy of the thermal; the latter has the dimension of acceleration and is expressed by the relation $b=g \rho^{\prime} /\langle\rho\rangle$, where $g$ is the acceleration of gravity, $\langle\rho\rangle$ is the unperturbed density of the surrounding medium, $\rho^{\prime}$ is the density perturbation; $N$ is the buoyancy frequency in the medium; $\beta$ and $\beta_{1}$ denote dimensionless constants, the former being determined by the rate of entrainment of the surrounding medium; in [2] the values $\beta=1 / 4$ and $\beta_{1}=2 / 3$ were considered.

The first equation of (1.1) describes the balance of mass of the moving thermal; it is assumed that the rate of entrainment of the surrounding medium is proportional to the surface area of the thermal and its velocity relative to the surrounding medium. We note that this equation is, generally speaking, written inaccurately — by implication, the right-hand side should obviously contain the absolute value of the thermal velocity relative to the surrounding medium rather than the velocity $w$. Generally speaking, the vertical velocity $w$ can also be negative (directed downward), for example, in the case of a thermal with negative buoyancy.

In [1] the problem is generalized to the important case where the thermal can move at an angle to the vertical. Such situations arise in a natural way when a thermal rises or descends in a horizontal flow with a vertical shift. For example, in the presence of significant convective instability in the atmosphere, the settling volume of air (a thermal with negative buoyancy) moving along the horizontal with fast background flow can transfer a significant horizontal momentum to the surface layer, in which the background horizontal flow is relatively slow, and this may be responsible for the occurrence of sharp gusts of wind - squall winds (microsqualls) in the surface layer.

As the thermal moves in such a flow, it also acquires a horizontal velocity relative to the background flow. Accordingly, the generalization of the first equation of (1.1) in [1] has the form

$$
\frac{d}{d t} R^{3}=3 \beta R^{2}\left(w^{2}+\Delta u^{2}\right)^{1 / 2} ; \quad \Delta u \equiv u-U .
$$

Here $u$ is the horizontal velocity component of the thermal, $U$ is the velocity of the background horizontal flow, which depends, generally speaking, on the height, and hence, on the time it takes for the thermal to move to a certain level. The system is also supplemented with the equation

$$
\frac{d}{d t}\left(R^{3} \Delta u\right)=-U^{\prime} R^{3} w
$$


where the prime denotes the vertical derivative (the shear of the velocity of the background flow; in this note it is assumed to be known and constant). This equation is analogous to the last equation of (1.1), but describes the change in the horizontal amount of motion.

To increase generality in the equation for changing buoyancy (the last equation of (1.1)), we additionally take into account the possibility of existence of the source of buoyancy in the thermal:

$$
\frac{d}{d t}\left(R^{3} b\right)=-N^{2} R^{3} w+Q .
$$

The term $Q$ is the intensity of the buoyancy source (connected, for example, with heat generation in the thermal [7]). Such problems with volumetric heat generation arise, in particular, in the theory of active influences on atmospheric processes. For artificial stimulation of convection, it is proposed to add to air, for example, a finely dispersed admixture absorbing short-wave solar radiation. A cloud of such an (almost weightless) admixture must ascend due to heat release. This convection is described using models such as thermals [7]. In the simplest cases, integral heat release in such a thermal can be taken to be constant: $Q=$ const.

The case of unstable background stratification of the surrounding medium, when $N^{2}<0$, was not considered in [1]. This case is of interest, in particular, in connection with the abovementioned mechanism of generation of microgusts in the atmosphere. It can be assumed that in an unstably stratified medium the vertically moving volume of the medium will accelerate. But in this case, entrainment intensifies and the volume of the thermal increases fast; consequently, its mobility must decrease. Therefore, there exists an important question: to what extent will the thermal in this case be carried away along the horizontal by the shear background flow will the thermal have time during vertical movement to "keep track" of the vertical shear of the background flow? It is this problem that is addressed in this note.

\section{Solution}

A closed system of equations is considered: the second equation of (1.1) and Eqs. (1.2), (1.3) and (1.4); the values of the sought-for quantities $R, u, w$ and $b$ at the initial time $t=0$ (marked below with a zero index) are taken as initial data. From the first and the last of the above-mentioned equations, passing in them to the variables $W(t)=R^{3} w, D(t)=R^{3} b$ and integrating, it is easy to obtain

$$
\begin{aligned}
R^{3} w & =A \exp (t / \tau)+B \exp (-t / \tau)-\beta_{1} \tau^{2} Q \\
R^{3} b & =[A \exp (t / \tau)-B \exp (-t / \tau)] / \beta_{1} \tau
\end{aligned}
$$

where $A$ and $B$ are constants of integration and $\tau=1 / \sqrt{\beta_{1}}|N|$. Taking into account the initial conditions, we have

$$
\begin{aligned}
& A=\frac{R_{0}^{3}}{2}\left(w_{0}+\beta_{1} \tau b_{0}+\beta_{1} \tau^{2} Q / R_{0}^{3}\right), \\
& B=\frac{R_{0}^{3}}{2}\left(w_{0}-\beta_{1} \tau b_{0}+\beta_{1} \tau^{2} Q / R_{0}^{3}\right) .
\end{aligned}
$$

Integrating (1.4), we obtain

$$
V(t) \equiv R^{3} \Delta u=\Delta u_{0} R_{0}^{3}-U^{\prime} \tau\left\{A[\exp (t / \tau)-1]-B[\exp (-t / \tau)-1]-\beta_{1} \tau Q t\right\} .
$$


Substituting (2.1) and (2.4) into (1.2) and integrating, we find

$$
R(t)=R_{0}\left\{1+\frac{4 \beta}{R_{0}^{4}} \int_{0}^{t}\left[W\left(t^{\prime}\right)^{2}+V\left(t^{\prime}\right)^{2}\right]^{1 / 2} d t^{\prime}\right\}^{1 / 4} .
$$

The problem is in principle solved. When $Q=0$, the integral in the last expression is in principle calculated analytically.

The integrand in (2.5) contains, in particular, the exponents $\exp (t / \tau)$ growing with time. On the time intervals $t \gg \tau$ the corresponding terms dominate, so that the integrand is proportional to $\exp (t / \tau)$. From here it is easy to obtain the asymptotics of the solution on large times.

\section{Analysis of an important particular case}

As an illustration we analyze the solution for the particular case $\Delta u_{0}=0, w_{0}=0, Q=0$. In this case, $A=-B=\beta_{1} R_{0}^{3} \tau b_{0} / 2$ and the expression (2.5) can be transformed to the form

$$
R(t)=R_{0}\left\{1+\frac{8 \beta \beta_{1} \tau b_{0}}{R_{0}} \int_{0}^{t}\left[\left(\operatorname{sh} \frac{t^{\prime}}{\tau}\right)^{2}+S\left(\operatorname{ch} \frac{t^{\prime}}{\tau}-1\right)^{2}\right]^{1 / 2} d t^{\prime}\right\}^{1 / 4} .
$$

Here the dimensionless number $S \equiv\left(\tau U^{\prime}\right)^{2}$ is close to the inverse Richardson number for background flow (up to sign and within a factor of the order of unity). The integral in the last expression is calculated analytically (it is convenient to use, for example, "Mathematica", an integrated system of computer mathematics); it can be represented as

$$
\tau \frac{\left[F_{1}(t / \tau) \operatorname{cth}(t / 2 \tau)-F_{2}(t / \tau)\right] F_{3}(t / \tau)}{F_{1}(t / \tau)},
$$

where

$$
\begin{aligned}
& F_{1}(t / \tau)=\{(1+S)[1-S+(1+S) \operatorname{ch}(t / \tau)]\}^{1 / 2}, \\
& F_{2}(t / \tau)=\sqrt{2} S \operatorname{csch}(t / 2 \tau) \ln \left[\sqrt{2}(1+S)^{1 / 2} \operatorname{ch}(t / 2 \tau)+F_{1}(t / \tau) /(1+S)^{1 / 2}\right], \\
& F_{3}(t / \tau)=\left\{S[-1+\operatorname{ch}(t / \tau)]^{2}+[\operatorname{sh}(t / \tau)]^{2}\right\}^{1 / 2} .
\end{aligned}
$$

As is easy to verify, in the limit $t \gg \tau$ this integral is approximately equal to $(1+S)^{1 / 2} \tau \exp (t / \tau) / 2$, whence

$$
R(t) \approx R_{0}\left\{1+\frac{4 \beta \beta_{1} \tau^{2} b_{0}}{R_{0}}(1+S)^{1 / 2} \exp (t / \tau)\right\}^{1 / 4}
$$

On the time intervals on which the thermal has time to increase noticeably $\left(R(t) \gg R_{0}\right)$, this yields the asymptotics

$$
\begin{aligned}
R(t) & \approx(1+S)^{1 / 8}\left(4 \beta \beta_{1} \tau^{2} b_{0} R_{0}^{3}\right)^{1 / 4} \exp (t / 4 \tau), \\
w(t) & \approx \frac{1}{4(1+S)^{3 / 8}}\left(\frac{\beta_{1} b_{0} R_{0}^{3}}{4 \beta^{3} \tau^{2}}\right)^{1 / 4} \exp (t / 4 \tau), \\
b(t) & \approx \frac{1}{4(1+S)^{3 / 8} \tau}\left(\frac{b_{0} R_{0}^{3}}{4 \beta^{3} \beta_{1}^{3} \tau^{2}}\right)^{1 / 4} \exp (t / 4 \tau), \\
\Delta u(t) & \approx-\frac{S^{1 / 2}}{4(1+S)^{3 / 8}}\left(\frac{\beta_{1} b_{0} R_{0}^{3}}{4 \beta^{3} \tau^{2}}\right)^{1 / 4} \exp (t / 4 \tau) .
\end{aligned}
$$


The coefficients at the exponents depend most strongly on the initial dimensions of the thermal. In particular, it follows from the last formula that the thermal does not keep up with the background flow when moving along the horizontal - the thermal systematically lags behind it when $w>0$ and $U^{\prime}>0$. What also follows from the last formula is a simple relation between two velocity components of the thermal relative to the surrounding medium: $\Delta u(t) / w(t) \approx-S^{1 / 2}$.

\section{Conclusion}

The nonlinear problem of the dynamics of a turbulent thermal in which stratification, the shear of the velocity of the background flow and heat release in the thermal are simultaneously taken into account can be solved in terms of quadratures. The solution describes, in particular, the nonlinear effect of interaction of the horizontal and vertical components of the motion of the thermal relative to the surrounding medium, since each of the components influences the rate of entrainment of the surrounding medium, i.e., the growth rate of the thermal size and hence its mobility. The solution in the case of unstable stratification of the medium has important specific features. The fact that a thermal is carried away by the shear flow only to a limited extent can be of practical importance in the context of transfer of substances in the presence of atmospheric convection.

\section{References}

[1] Ingel, L. Kh., Nonlinear Dynamics of Turbulent Thermals in Shear Flow, J. Appl. Mech. Tech. Phys., 2018, vol. 59, no. 2, pp. 206-211; see also: Prikl. Mekh. Tech. Phys., 2018, no. 4, pp. 23-30.

[2] Turner, J. S., Buoyancy Effects in Fluids, Cambridge: Cambridge Univ. Press, 1973.

[3] Andreev, V. and Panchev, S., Dynamics of Atmospheric Thermals, Leningrad: Hydrometeoizdat, 1975 (Russian).

[4] Scorer, R. S., Environmental Aerodynamics, New York: Horwood, 1978.

[5] Gostintsev, Yu. A., Lazarev, V.V., Solodovnik, A.F., and Shatskih, Yu.V., Turbulent Termal in Stratified Atmosphere, Fluid Dyn., 1986, vol.21, no.6, pp.965-976; see also: Izv. Akad. Nauk SSSR Mekh. Zhidk. Gaza, 1986, no. 6, pp. 141-153.

[6] Emanuel, K.A., Atmospheric Convection, Oxford: Oxford Univ. Press, 1994.

[7] Ingel', L. Kh., Self-Action of Heat-Releasing Admixtures in a Liquid Medium, Physics-Uspekhi, 1998, vol. 41, no. 1, pp. 95-99; see also: Uspekhi Fiz. Nauk, 1998, vol. 168, no. 1, pp. 104-108. 